\title{
COMPARTMENTAL MODELING APPROACH OF LOSARTAN TRANSDERMAL TRANSPORT IN VITRO
}

\author{
Akhmad Kharis Nugroho ${ }^{1}$, Annas Binnarjo ${ }^{1,2}$, Arief Rahman Hakim ${ }^{1}$, Yunita Ermawati ${ }^{1}$
} Universitas Gadjah Mada, Sekip Utara Yogyakarta, 55281 Indonesia

2Faculty of Pharmacy Universitas Ahmad Dahlan, Jl. Prof. Supomo Janturan Umbulharjo, 55237 Yogyakarta

Submitted: $19-11-2013$

Accepted: $28-12-2013$

*Corresponding author Akhmad Kharis Nugroho

Email :

a.k.nugroho@ugm.ac.id
1Faculty of Pharmacy

Revised: $04-12-2013$

\begin{abstract}
Development of losartan in transdermal formulation is important due to its low oral bioavailability. Optimal transdermal formulation requires transport mechanism understanding. This study was aimed to develop and evaluate compartmental modeling of losartan transdermal transport in vitro to fulfil this demand. Losartan solution $(10 \mathrm{~g} / \mathrm{L}$ in $20 \%$ propylene glycol) was filled into the acceptor phase of a vertical diffusion-cells system. The fresh rat skin (pretreated with oleic acid for 1 hour) was used as the transport membrane separating the donor and the acceptor phase, which was filled with phosphate-buffered-saline $\mathrm{pH}$ 7.4. During $30 \mathrm{~h}$, samples were collected and analyzed using HPLC method. Four compartmental models were proposed, i.e. models with one (model 1) or two (model 2) lag-compartment(s) either with a zero-order (model A) or a first- order (model B) drug input from donor to the skin. WinSAAM was used to evaluate the models based on the parameters: 1) visual goodness of fit (GOF); and 2) Corrected Akaike's Information Criterion $(A I C C)$. Both the GOF and AICC evaluations indicated model 2-A as the best model describing losartan transdermal transport. The model suggested that after reaching the upper layer of skin at a constant rate, losartan transport was split into two flows. Both flows are transited in two separate lagcompartments before reaching into the acceptor phase.
\end{abstract}

Key words: Transdermal, Losartan, Modeling, Lag-compartment, WinSAAM

\section{INTRODUCTION}

Losartan, an angiotensin II type 1 receptor antagonist (Goldberg et al., 1995), has been used in hypertension therapy since 1995, and becoming the drug of choice particularly for diabetes patients (Maggon, 2009). It is marketed in per-oral dosage form as a tablet with a dose of $50 \mathrm{mg}$. Nevertheless, losartan is intensively metabolized in the hepatic system (first pass effect) as well as metabolized by the normal flora in the gut. These processes are responsible for a low oral bioavailability of the drug (approximately 33\%) (Lo et al., 1995). Moreover, losartan has also a relatively fast elimination rate from the body (elimination half life of $1.5-2.5$ h) (Ohtawa et al., 1993).

Those situations suggested the significance of an alternative of drug delivery system, circumventing such problems, with transdermal delivery system as one of good candidate (Nugroho, 2005). Transdermal delivery of losartan could deliver the drug across the skin to reach systemic circulation in the dermis layer in a non invasive manner. The delivery method can by-pass metabolism in the hepatic and in the gut. It is therefore expected that delivery of drugs via such route can increase their bioavailability (Nugroho, 2005).

Currently there are a limited number of studies focusing on transdermal transport of losartan. Petkar and Kuchekar examined the influence of capsaicin in transdermal transport of losartan (Petkar and Kuchekar, 2007). The use of proniosomes to facilitate transdermal transport of losartan has also been reported by Thakur and coworker (Thakur et al., 2009).

Furthermore, none of those studies were concerned on the transport mechanism of transdermal delivery of losartan. Such investigation is important in order to shed light on how drug molecules behave during the 
transport across the skin layer. One approach to study such mechanism is based on development of compartmental model to describe transdermal delivery transport.

Such approach has been started in 1982 by Guy and coworker who proposed a series of compartmental models on transdermal transport of several drugs (Guy et al., 1982). Nugroho et al proposed a series of compartmental models to describe drug transport by transdermal iontophoresis in vitro (Nugroho et al., 2004) and in vivo (Nugroho et al., 2005).

The models were developed and implemented using Winnonlin (Nugroho et al., 2005, 2004) as well as NONMEM (Nugroho et al., 2006), well-known examples of commercial software used in pharmacokinetics-pharmacodynamics modeling.

Later on, Nugroho (2008) proposed a modeling approach on the same focus using WinSAAM, a free software widely used for biological system modeling developed under support of National Insitute of Health USA (Stefanovski et al., 2003). In this approach, several basic transdermal transport models were developed, implementing a transport flow of drug from the donor or patch via the skin before entering into the acceptor phase or plasma (Nugroho, 2008).

The aim of the current study was to develop and evaluate compartmental modeling approach which could explain the mechanism of transdermal transport in vitro of losartan. The proposed models implement one or two lag compartment(s) between donor and skin to describe transport data.

\section{MATERIAL AND METHODS}

Losartan potassium was obtained from PT Kalbe Farma Jakarta Indonesia. Propylene glycol and oleic acid (pharmaceutical grades) were purchased from PT Brataco Chemicals, Yogyakarta Indonesia. Phosphate buffered saline was prepared by mixing dan dissolving ingredients i.e.: bi-sodium hydrogen phosphate $\left(\mathrm{Na}_{2} \mathrm{HPO}_{4}\right)$, Sodium di-hydrogen Phosphate $\left(\mathrm{NaH}_{2} \mathrm{PO}_{4}\right)$ and sodium chloride (all are in analytical grade (Merck, Darmstadt Germany) and purchased from PT General Labora Yogyakarta).

\section{Rat skin preparation}

Fresh rat skin was obtained directly after animal sacrifice using a lethal dose of ether inhalation method. Skin was then carefully cleaned from its hair by electric clipping without any damage, wound or scratch on the skin surface. Skin fat was removed by using scalpel. Thereafter, skin was cleaned with double distillated water prior to cutting into circular shapes using surgery scissors with a diameter of $2 \mathrm{~cm}$. Skin was then putted onto the diffusion cell, either for pretreatment studies or directly for a transport study.

\section{Skin pretreatment}

After being mounted into diffusion cells, skin was exposed to oleic acid (used as the permeation enhancer), which was filled into the donor compartment of the diffusion cells. The skin pretreatment was performed for 1 hour. After the pretreatment, the enhancer was removed from the cells and skin was then cleaned by soaking and spraying with double distilled water 5 times. Thereafter skin was ready for the transport study.

\section{Transport study}

The transport studies were performed in the vertical type diffusion cells, prepared by Laboratory of Material Process, Department of Physical Engineering Institut Teknologi Bandung, Bandung Indonesia. Losartan donor solution was prepared at a concentration of $10 \mathrm{~g} / \mathrm{L}$ (in $20 \%$ propylene glycol in $0.05 \mathrm{M}$ citric buffer $\mathrm{pH}$ 5). After proper mounting of the rat skin, the cells system was then secured by joining and screwing the donor and the acceptor parts together. The donor phase of the system was then filled in with losartan solution. The acceptor phase was filled in with $0.15 \mathrm{M}$ phosphate buffer saline at $\mathrm{pH}$ 7.4. The transport study was performed for 30 hours, during which, samples were collected periodically from the opening column on the acceptor phase side.

\section{HPLC analyses}

Quantitative analyses were performed based on the HPLC method (HPLC Prominence Shimadzu, Kyoto, Japan). Briefly, the eluent consisted of $0.05 \mathrm{~N}$ acetic 


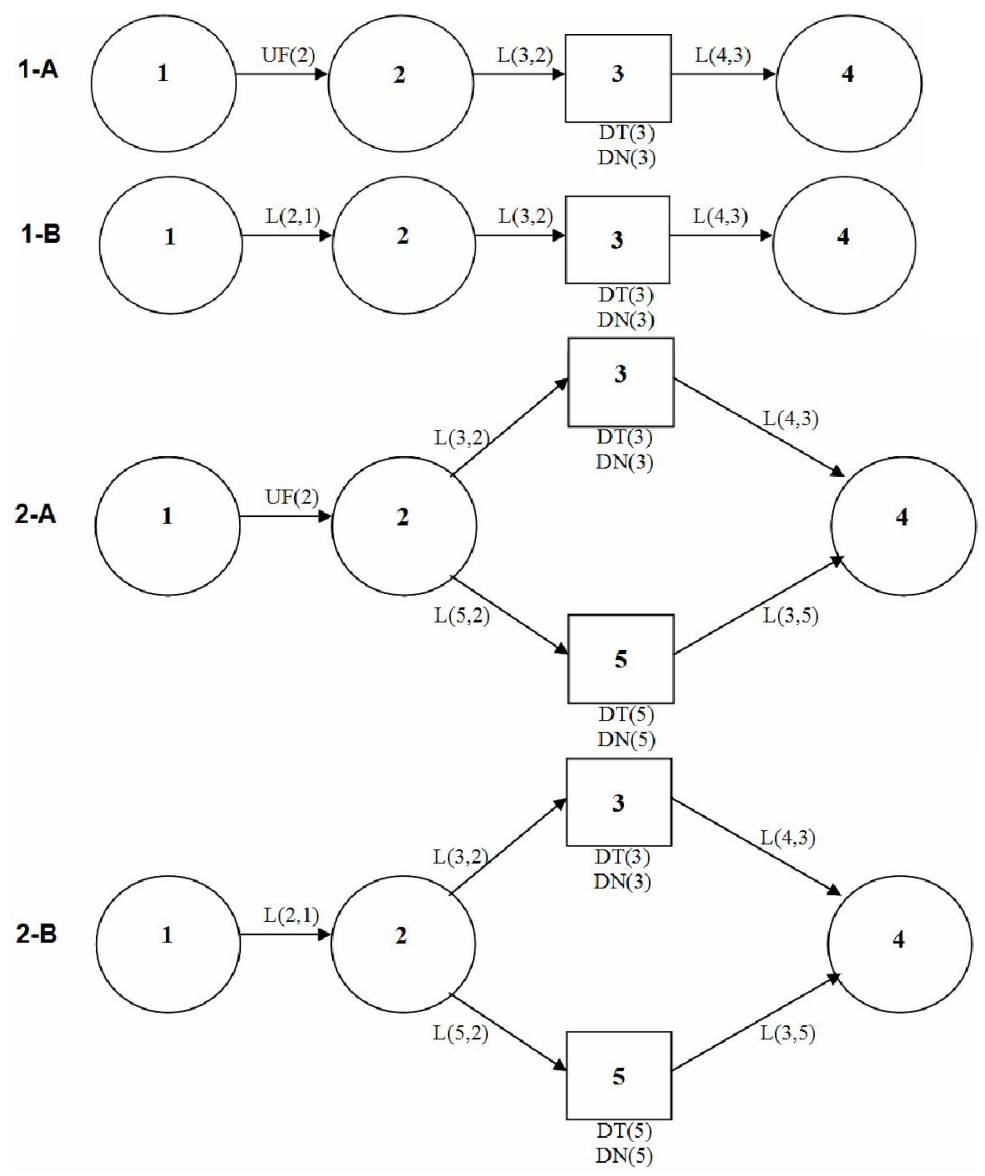

Figure 1. Schematic Compartmental models for transdermal transport of losartan using WinSAAM.

buffer and acetonitrile with a volume ratio of 60:40 and pumped into a LichroCART 250-4 RP $18(5 \mu \mathrm{m})$ (Merck, Darmstadt Germany) at a flow rate of $0.75 \mathrm{~mL} / \mathrm{min}$. The detection was performed using ultraviolet detector (SPD $20 \mathrm{~A}$ Shimadzu, Kyoto, Japan) at a wavelength of $223 \mathrm{~nm}$.

\section{Modeling analyses}

The cumulative amounts of the transdermal transport of losartan were plotted as a function of time. Data were then analyzed based on the compartmental model approach. In this approach, compartmental models consisted of models with one (model 1) or two (model 2) lag-compartment(s) either with a zero-order (model A) or a first- order (model B) drug input from donor to the skin. Therefore, there were four compartmental models used i.e. model 1-A, model 1-B, model 2-A and model 2-B.

The modeling was performed using WinSAAM. The flow of mass transfer in each model, implemented the notation rule in WinSAAM, is depicted in figure 1 . In accordance to this respect, model parameters were defined, and there are several important notations as follows: 1) IC(1) i.e. initial dose amount (in the donor phase) available for a first order transport; 2$) L(y, x)$ i.e. coefficient of drug transport from the compartment $x$ to the compartment $y$ (i.e. $L(2,1), \quad L(3,2), L(4,3)$, $L(5,2)$, and $L(4,5))$; 3)UF(2) i.e. a zero order (constant) of drug transport rate into compartment 2 (stratum corneum); 4) DT(z) i.e. lag or delay time in compartment z (i.e DT(3) and $D T(5))$; and 5) $D N(₹)$ i.e. theoretical delay number of partial elements or pseudo- 
I

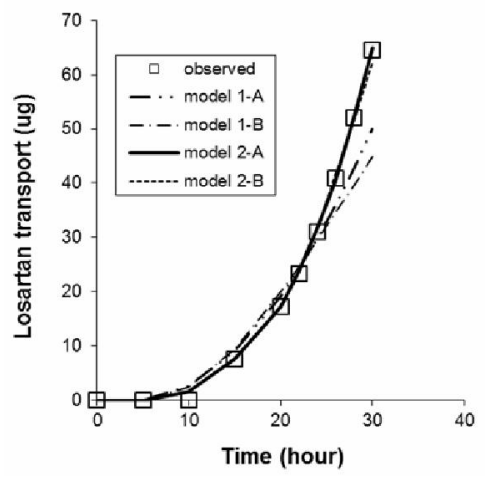

II

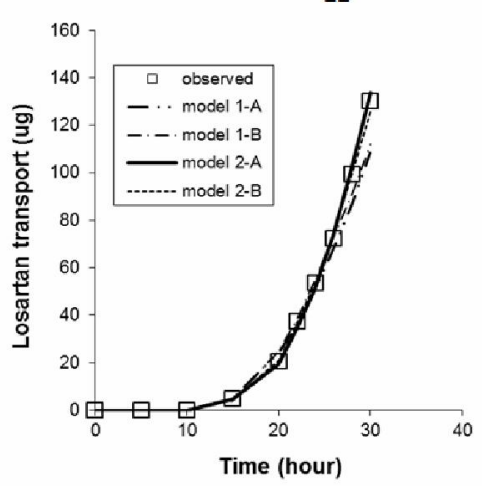

III

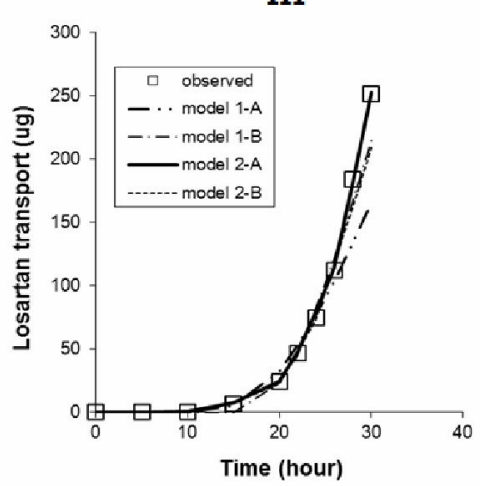

Figure 2. Observed data of transdermal transport of losartan of 3 samples in transport studies, presented together with the model prediction using model 1-A (-..-), model 1-B (-.-.), model 2-A (-- ) and model 2-B (- - -)

compartments in a certain lag compartment z. (i.e. $D N(3)$ and $D N(5)$ ). Furthermore, all of those parameters together with the data were built into a WINSAAM listing, which then being executed using a normal WinSAAM command i.e. deck, solve and iter. Evaluation of model appropriateness to transport data was performed based on the evaluation of: 1) visual goodness of fit $(G O F)$; and 2) Corrected Akaike's Information Criterion (AICc) (Motulsky and Christopoulos, 2003).

\section{RESULTS AND DISCUSSION}

\section{Transdermal transport of losartan}

Losartan was rather slowly permeated across the skin. It needs several hours before being detected in a significant amount in the acceptor phase. For this reason, study transport was performed for up to $30 \mathrm{~h}$. The typical profiles of transdermal transport of losartan in this study are presented in figure 2 .

There are several possible explanations on the phenomenon. As the study used full thickness rat skin, most likely the thickness of the membrane retained drug molecules longer and release the drug much slower. Moreover, the condition could also be due to a slow movement of losartan across the epidermis and dermis layer in the rat skin. According to SRC PhysProp Database and DrugBank database, losartan $\log \mathrm{P}$ was estimated at a value of 4.01 (Anonymous, 2013a) and 6.1 (Anonymous, 2013b), respectively. These data suggest losartan as a very lipophilic compound. Such situation might result in difficulties for losartan to pass through an aqueous environment in the skin.

\section{Modeling of transdermal transport of losartan}

The results of the compartmental model of transdermal transport of losartan are presented in figure 2 (presentation of model fitting) and figure 3 (model evaluation based on the standard goodness of fit evaluations, i.e. 1) the correlation of $Q O$ (observed transport of losartan) versus $Q C$ (predicted transport of losartan) presented together with line of identity (panels a); 2) the correlation of residual versus time (panels b); and 3) the correlation of residual versus $Q O$ (panels c)).

Data in figure 2 showed model 2-A could describe the observation data better than other models. Such indication was in agreement with data on figure 3 , where the goodness of fit evaluations also indicated the less deviation between model prediction and the observed data.

Further evaluation was performed based on the AICc calculation (Motulsky and Christopoulos, 2003). The results of $A I C c$ parameters calculations are presented in table I. According to the recent approach, model with the lowest value of $A I C c$ value should describe the observed data better than the other models. Data in table I demonstrate that Model 2-A has 

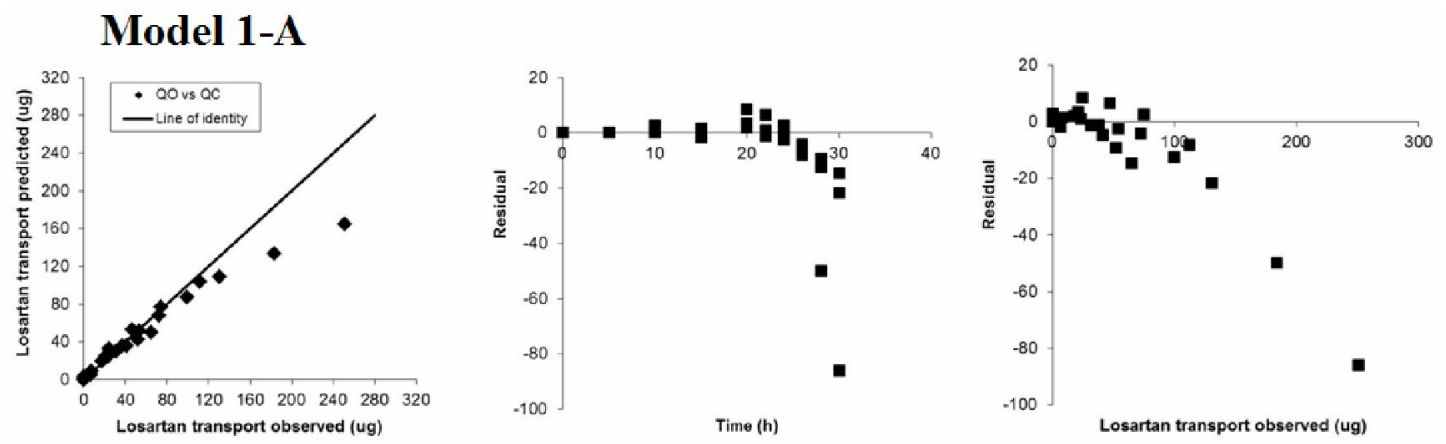

Model 1-B
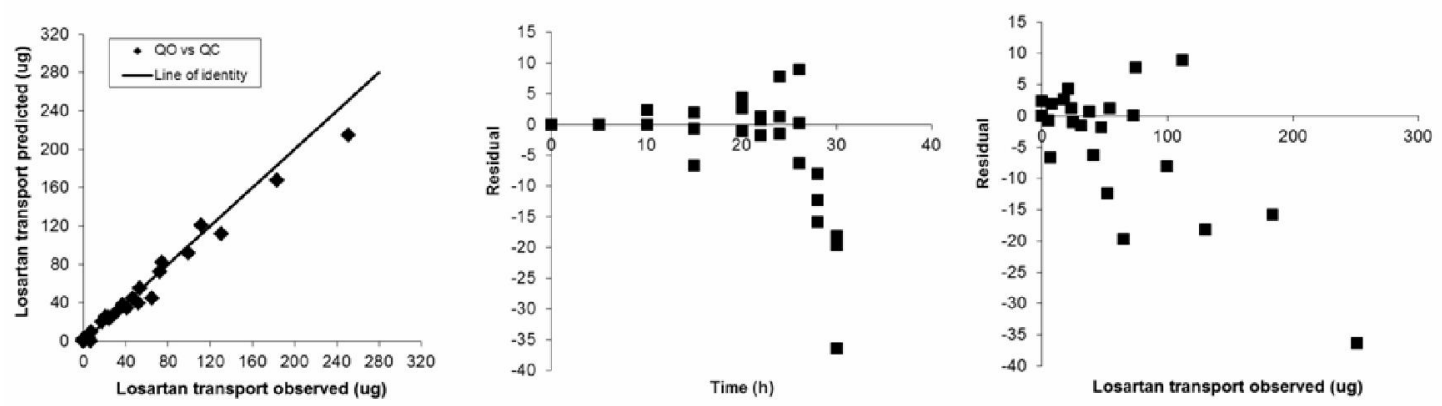

Model 2-A
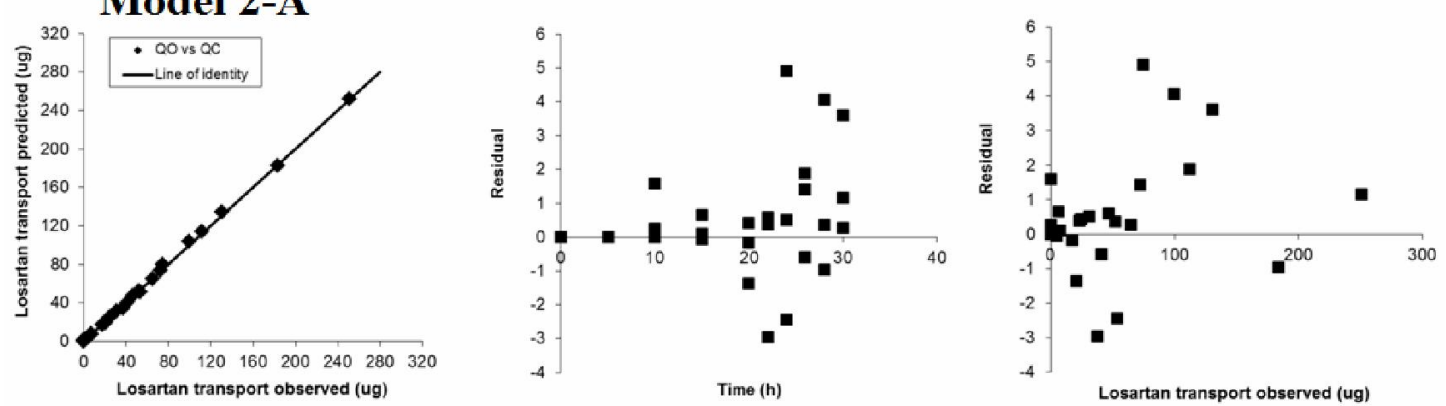

Model 2-B
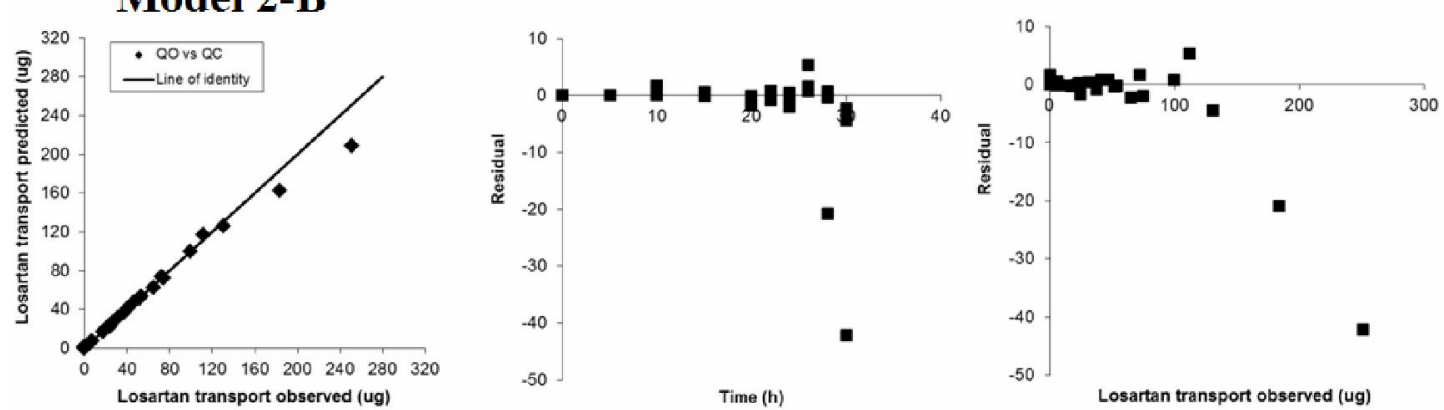

Figure 3. Typical examples of goodness of fit (GOF) of transdermal iontophoresis of losartan (sample no II) fitted using model 1-A, 1-B, 2-A, and 2-B. Panels a depict GOF based on the correlation of QO (observed transport of losartan) versus QC (predicted transport of losartan) presented together with line of identity (solid line). Panels $\mathbf{b}$ and $\mathbf{c}$ respectively depict GOF evaluations based on correlation of residual versus time, and correlations of residual versus QO. 
Table I. AICc Parameters of model 1-A, 1-B, 2-A and 2-B to describe transdermal transport of losartan

\begin{tabular}{ccccc}
\hline Replicate & Model 1-A & Model 1-B & Model 2-A & Model 2-B \\
\hline 1 & 33.89 & 79.43 & -121.97 & -82.74 \\
2 & 25.48 & 72.36 & -107.38 & -86.07 \\
3 & 85.03 & 95.81 & -101.97 & -61.42 \\
\hline
\end{tabular}

the lowest value of $A I C c$. Thus, the $A I C c$ analyses also suggested model $2-\mathrm{A}$ as the best model to properly describe transdermal transport of losartan.

Moreover, it is very interesting to observe that transdermal transport of losartan was best described using model 2-A. As presented in figure 1 , the model implements 2 separate lag compartments (compartments 3 and 5) after the outer part of skin (stratum corneum, compartment 2). The presence of both lag compartments may retain drug molecules longer before entering into the acceptor compartment (compartment 4 in Figure 1). Surprisingly, it is in agreement with the analyses that due to its high lipophilicity, losartan has difficulties to pass through the hydrophilic parts of the skin, i.e. epidermis and dermis. Thus, most likely, the lag compartments are present in these parts of skin.

Moreover, there are several possible explanations why there are separate lag compartments in this transport. First, losartan is present in 2 forms, either as unionized or as ionized states. Values of skin $\mathrm{pH}$ were reported in several studies, briefly it was in the range of 4 to 6 (Plasencia et al., 2007). As the pKa of losartan is 4.9 (Pedroso et al., 2009), based on Henderson-Hasselbalch equation, losartan will be approximately in 50:50 fractions of unionized and ionized forms. According to calculation using Marvin software (ChemAxon, 2013), the $\log \mathrm{P}$ of ionized losartan is 0.49 while in the unionized form its value is estimated at 5.08. Thus the ionic form will have difficulties to cross the stratum corneum while the unionized form should have difficulties to transport across the hydrophilic part of the skin (epidermis and dermis).

In contrast, the proposed models (model 2-A and 2-B) indicated the lag compartment were present somewhere after the outer part of skin. It seems to be controversial that the model indicated even for the ionized part of losartan, the lag compartment is not located on the stratum corneum itself. Such confusion should be solved by considering the skin pretreatment using oleic acid as well as the presence of propylene glycol in the donor solution. Combination of oleic acid and propylene glycol are well known to have enhancement effect on transdermal transport of several compound. In this respect, stratum corneum lipids could become perturbed, which result in an easier drug passage across this outermost layer of skin (Larrucea et al., 2001).

The remaining question on why there are 2 lag compartments after the stratum corneum could be answered by considering the fact of the excised rat skin. As the epidermis and dermis layers of the living skin contain a lot of water, when skin has been excised from the body, the water turn into a stagnant condition. This, in fact, could present as the barrier retaining the drug molecules, and functioning as a lag compartment. There are 2 lag compartments, as the ionized and unionized losartan may have different challenges to pass through the stagnant layers. The ionized part, due to higher solubility in water, could pass through the dermis and dermis easier than the unionized one. Thus, the unionized part of losartan stays longer than the ionized one, which result in a different duration of lag time.

It is clear that, the proposed compartment model, i.e 2-A, could explain the transport data, and even to explain how the mechanism of drug transport across stratum corneum. Moreover, although more studies will be required to fully shed light on the mechanism of transdermal transport across the skin, the finding of the research strongly suggested that the proposed compartmental model could provide insight into the mechanism which is difficult to be solved using other methods. 


\section{CONCLUSION}

In conclusion, the investigation indicates that the compartmental modeling approach appropriately describe transdermal transport of losartan in vitro. The model could also be used to determine losartan transport mechanism.

\section{ACKNOWLEDGEMENT}

The research was supported by Hibah Kompetensi DIKTI batch II from The Ministry of Education Council Republic of Indonesia, year 2009-2011.

\section{REFERENCES}

Anonymous, 2013a. Data from SRC PhysProp database [WWW Document]. URL http://esc.syrres.com/interkow/webpro p.exe?CAS $=114798-26-4 \quad$ (accessed 11.29.13).

Anonymous, 2013b. DrugBank: Showing drug card for losartan (DB00678) [WWW Document]. URL http:/ /www.drugbank.ca/drugs/DB006 78 (accessed 11.29.13).

ChemAxon, 2013. Marvin - draw, view \& visualize structure, query \& reaction «ChemAxon-cheminformatics platforms and desktop applications [WWW Document]. URL http://www. chemaxon.com/products/marvin/ (accessed 12.23.13).

Goldberg, M.R., Bradstreet, T.E., McWilliams, E.J., Tanaka, W.K., Lipert, S., Bjornsson, T.D., Waldman, S.A., Osborne, B., Pivadori, L., Lewis, G., 1995. Biochemical effects of losartan, a nonpeptide angiotensin II receptor antagonist, on the renin-angiotensinaldosterone system in hypertensive patients. Hypertension, 25, 37-46.

Guy, R.H., Hadgraft, J., Maibach, H.I., 1982. A pharmacokinetic model for percutaneous absorption. Int J Pharm.,11, 119 - 129.

Larrucea, E., Arellano, A., Santoyo, S., Ygartua, P., 2001. Combined effect of oleic acid and propylene glycol on the percutaneous penetration of tenoxicam and its retention in the skin. Eur J Pharm Biopharm 52, 113-119.

Lo, M., Goldberg, M., Mccrea, J., Lu, H., Furtek, C., Bjornsson, T., 1995. Pharmacokinetics of losartan, an angiotensin II receptor antagonist, and its active metabolite EXP3174 in humans. Clin Pharmacol Ther., 58, 641649.

Maggon, K., 2009. Global angiotensin II receptor antagonists market review 2008 - a knol [WWW Document]. URL http:/ / knol.google.com/k/globalangiotensin-ii-receptor-antagonistsmarket-review-2008\# (accessed 12.16.09).

Motulsky, H.J., Christopoulos, A., 2003. Fitting models to biological data using linear and nonlinear regression. A practical guide to curve fitting. GraphPad Software, Inc., San Diego CA.

Nugroho, A.K., 2005. Transdermal iontophoretic delivery of dopamine agonists: In vitro - In vivo correlation based on novel compartmental modeling. Ph.D thesis, Leiden University, Leiden, The Netherlands.

Nugroho, A.K., 2008. Modeling transpor obat secara transdermal in vitro menggunakan software WinS AAM: Kinetika orde nol dan orde satu, in: Prosiding Kongres Ilmiah XVI ISFI 2008. Ikatan Sarjana Farmasi Indonesia, Yogyakarta Indonesia, pp. 845 $-852$.

Nugroho, A.K., Della Pasqua, O., Danhof, M., Bouwstra, J.A., 2004. Compartmental modeling of transdermal iontophoretic transport: I. In vitro model derivation and application. Pharm Res., 21, 1974-1984.

Nugroho, A.K., Della-Pasqua, O., Danhof, M., Bouwstra, J.A., 2005. Compartmental modeling of transdermal iontophoretic transport II: in vivo model derivation and application. Pharm Res., 22, 335-346.

Nugroho, A.K., Romeijn, S.G., Zwier, R., de Vries, J.B., Dijkstra, D., Wikström, H., Della-Pasqua, O., Danhof, M., Bouwstra, J.A., 2006. Pharmacokinetics and pharmacodynamics analysis of transdermal iontophoresis of 5-OHDPAT in rats: in vitro-in vivo correlation. $J$ Pharm Sci., 95, 1570-1585.

Ohtawa, M., Takayama, F., Saitoh, K., Yoshinaga, T., Nakashima, M., 1993. Pharmacokinetics and biochemical efficacy after single and multiple oral 
administration of losartan, an orally active nonpeptide angiotensin II receptor antagonist, in humans. Br J Clin Pharmacol., 35, 290-297.

Pedroso, C., de Oliveira, J., Campos, F., Gonçalves, A., Trindade, A., Pontarolo, R., 2009. A Validated RP-LC Method for Simultaneous Determination of Losartan Potassium and Amlodipine Besilate in Pharmaceutical Preparations. Chromatographia, 69, 201-206.

Petkar, K.C., Kuchekar, S.., 2007. In-vitro percutaneous absorption of losartan potassium in human skin and prediction of human skin permeability. $D A R U, \mathbf{1 5}$, $53-60$.
Plasencia, I., Norlén, L., Bagatolli, L.A., 2007. Direct visualization of lipid domains in human skin stratum corneum's lipid membranes: effect of $\mathrm{pH}$ and temperature. Biophys J., 93, 3142-3155.

Stefanovski, D., Moate, P.J., Boston, R.C., 2003. WinSAAM: a windows-based compartmental modeling system. Metab Clin Exp. 52, 1153-1166.

Thakur, R., Anwer, M.K., Shams, M.S., Ali, A., Khar, R.K., Shakeel, F., Taha, E.I., 2009. Proniosomal transdermal therapeutic system of losartan potassium: development and pharmacokinetic evaluation. J Drug Target, 17, 442-449. 